\title{
Non-Fermi-liquid behavior in the Kondo lattices induced by peculiarities of magnetic ordering and spin dynamics
}

\author{
V.Yu.Irkhin* and M.I.Katsnelson \\ Institute of Metal Physics, 620219 Ekaterinburg, Russia
}

\begin{abstract}
A scaling consideration of the Kondo lattices is performed with account of singularities in the spin excitation spectral function. It is shown that a nonFermi-liquid (NFL) behavior between two critical values of the bare $s-f$ coupling constant occurs naturally for complicated magnetic structures with several magnon branches. This may explain the fact that a NFL behavior takes place often in the heavy-fermion systems with peculiar spin dynamics. Another kind of a NFL-like state (with different critical exponents) can occur for simple antiferromagnets with account of magnon damping, and for paramagnets, especially with two-dimensional character of spin fluctuations. The mechanisms proposed lead to some predictions about behavior of specific heat, resistivity, magnetic susceptibility, and anisotropy parameter, which can be verified experimentally.
\end{abstract}

Typeset using REVTEX 


\section{INTRODUCTION}

Recently, a great experimental material has been obtained for anomalous $f$-systems demonstrating so-called non-Fermi-liquid (NFL) behavior [1,2]. Manifestations of the NFL behavior are unusual temperature dependences of magnetic susceptibility $(\chi(T) \sim$ $\left.T^{-\zeta}, \zeta<1\right)$, electronic specific heat $\left(C(T) / T\right.$ is proportional to $T^{-\zeta}$ or $\left.-\ln T\right)$ and resistivity $\left(\rho(T) \sim T^{\mu}, \mu<2\right)$, etc. Such a behavior is observed not only in alloys where disorder is present $\left(\mathrm{U}_{x} \mathrm{Y}_{1-x} \mathrm{Pd}_{3}, \mathrm{UPt}_{3-x} \mathrm{Pd}_{x}, \mathrm{UCu}_{5-x} \mathrm{Pd}_{x}, \mathrm{CeCu}_{6-x} \mathrm{Au}_{x}, \mathrm{U}_{x} \mathrm{Th}_{1-x} \mathrm{Be}_{13}\right)$, but also in some stoichiometric compounds, e.g., $\mathrm{Ce}_{7} \mathrm{Ni}_{3}$ [4], $\mathrm{CeCu}_{2} \mathrm{Si}_{2}, \mathrm{CeNi}_{2} \mathrm{Ge}_{2}$ [5]. The latter situation is most interesting from the physical point of view [6]. There are a number of theoretical mechanisms proposed to describe the NFL state: two-channel Kondo scattering [7,8,9], "Griffiths singularities" in disordered magnets [10,11, strong spin fluctuations near a quantum magnetic phase transition [12,13,14 etc. Most of modern treatments of the NFL problem have a semiphenomenological character. The only microscopic model where formation of the NFL state is proven - the one-impurity two-channel Kondo model - seems to be insufficient, since important role of intersite interactions is now a matter of common experience [1].

In the present paper we start from the standard microscopic model of a periodical Kondo lattice. Main role in the physics of the Kondo lattices belongs to the interplay of the on-site Kondo screening and intersite exchange interactions. This interplay results in the mutual renormalization of the characteristic energy scales: the Kondo temperature $T_{K}$ and spinfluctuation frequency $\bar{\omega}$. We shall demonstrate that during the renormalization process "soft" boson branches can be formed, presence of singularities of spin spectral function being of crucial importance. Scattering of electrons by such soft collective excitations just leads to the formation of the NFL state (cf. [14]).

In Sect. 2 the renormalization group (scaling) equations are presented. In Sect.3 we consider the antiferromagnetic (AFM) state with account of the spin-wave damping and the paramagnetic state with simple spin-diffusive dynamics. It turns out that in these cases a 
NFL-like behavior (in a restricted temperature interval) is possible, especially in the case of quasi-two-dimensional (2D) spin fluctuations. In Sect. 4 we consider the general problem of singularities of the scaling function. In Sect.5 we show that the NFL behavior up to lowest temperatures can be naturally obtained provided that we take into account magnon-like excitations in the case of a complicated spin dynamics. The excitation picture required is characteristic for real $f$-systems where several excitation branches exist. In Sect. 6 we discuss various physical properties and possible relation to experimental data.

\section{THE SCALING EQUATIONS}

To describe a Kondo lattice, we use the $s-f$ exchange model

$$
H=\sum_{\mathbf{k} \sigma} t_{\mathbf{k}} c_{\mathbf{k} \sigma}^{\dagger} c_{\mathbf{k} \sigma}-I \sum_{i \alpha \beta} \mathbf{S}_{i} \boldsymbol{\sigma}_{\alpha \beta} c_{i \alpha}^{\dagger} c_{i \beta}+\sum_{\mathbf{q}} J_{\mathbf{q}} \mathbf{S}_{-\mathbf{q}} \mathbf{S}_{\mathbf{q}}+H_{a}
$$

where $t_{\mathbf{k}}$ is the band energy, $\mathbf{S}_{i}$ and $\mathbf{S}_{\mathbf{q}}$ are spin-density operators and their Fourier transforms, $I$ is the $s-f$ exchange parameter, $J_{\mathbf{q}}$ are the intersite exchange parameters, $\sigma$ are the Pauli matrices, $H_{a}$ is the anisotropy Hamiltonian which results in occurrence of the gap $\omega_{0}$ in the spin-wave spectrum. In Refs. [15, 16], the interplay of the Kondo effect and intersite interactions was investigated by the renormalization group approach. The latter starts from the second-order perturbation theory with the use of the equation-of-motion method (within the diagram technique in the pseudofermion representation for the spin operators, such an approximation corresponds to the one-loop scaling). The relevant variables are the effective (renormalized) parameter of $s-f$ coupling $g_{e f}(C)=-2 \rho I_{e f}(C)(C \rightarrow-0$ is a flow cutoff parameter, $\rho$ is the bare density of electron states at the Fermi level), characteristic "exchange" spin-fluctuation energy $\bar{\omega}_{e x}(C)$, gap in the spin-wave spectrum $\omega_{0}(C)$, and magnetic moment $\bar{S}_{e f}(C)$. In the magnetically ordered phase, $\bar{\omega}_{e x}$ is the magnon frequency $\omega_{\mathbf{q}}$, which is averaged over the wavevectors $\mathbf{q}=2 \mathbf{k}$ where $\mathbf{k}$ runs over the Fermi surface; in the case of dissipative spin dynamics (paramagnetic phase) $\bar{\omega}_{e x}$ is determined by the second moment of the spin spectral density. 
Here we write down the set of scaling equations with account of the magnon damping $\bar{\gamma}(C)$ :

$$
\begin{aligned}
\partial g_{e f}(C) / \partial C & =\Lambda, \partial \ln \bar{S}_{e f}(C) / \partial C=-\Lambda / 2 \\
\partial \ln \bar{\omega}_{e x}(C) / \partial C & =-a \Lambda / 2, \partial \ln \omega_{0}(C) / \partial C=-b \Lambda / 2 \\
\partial \ln \bar{\gamma}(C) / \partial C & =-c \Lambda / 2
\end{aligned}
$$

with

$$
\Lambda=\Lambda\left(C, \bar{\omega}_{e x}(C), \omega_{0}(C)\right)=\frac{g_{e f}^{2}(C)}{|C|} \eta\left(\frac{\bar{\omega}_{e x}(C)}{|C|}, \frac{\omega_{0}(C)}{|C|}, \frac{\bar{\gamma}(C)}{|C|}\right)
$$

$a=1-\alpha$ for the paramagnetic (PM) phase, $a=1-\alpha^{\prime}, b=1$ for the antiferromagnetic $(\mathrm{AFM})$ phase, $a=2\left(1-\alpha^{\prime \prime}\right), b=2$ for the ferromagnetic (FM) phase; $\alpha, \alpha^{\prime}, \alpha^{\prime \prime}$ are some averages over the Fermi surface (see Ref. [15]). For the staggered AFM ordering in the 2D and 3D cubic lattices where

$$
J_{\mathbf{q}}=2 J_{1} \sum_{i=1}^{d} \cos q_{i}+4 J_{2} \sum_{i>j}^{d} \cos q_{i} \cos q_{j}
$$

with $J_{1}$ and $J_{2}$ being the exchange integrals between nearest and next-nearest neighbors $\left(\left|J_{1}\right| \gg\left|J_{2}\right|\right)$, one can derive by using expansion in small $q$

$$
\alpha^{\prime} \simeq 2(d-1) \frac{J_{2}}{J_{1}}\left|\left\langle\exp \left(i \mathbf{k} \mathbf{R}_{2}\right)\right\rangle_{t_{\mathbf{k}}=0}\right|^{2}
$$

where $\mathbf{R}_{2}$ runs over the next-nearest neighbors, the electron spectrum $t_{\mathbf{k}}$ is referred to the Fermi energy $E_{F}=0$. Similar equations can be obtained for the Coqblin-Schrieffer model [15] and in the case of anisotropic $s-f$ coupling [16].

Using (2)-(4) we obtain the explicit expressions

$$
\begin{aligned}
\bar{\omega}_{e x}(C) & =\bar{\omega}_{e x} \exp \left(-a\left[g_{e f}(C)-g\right] / 2\right) \\
\omega_{0}(C) & =\omega_{0} \exp \left(-b\left[g_{e f}(C)-g\right] / 2\right) \\
S_{e f}(C) & =S \exp \left(-\left[g_{e f}(C)-g\right] / 2\right) \\
\bar{\gamma}(C) & =k g_{e f}^{2}(C) \bar{\omega} \exp \left(-c\left[g_{e f}(C)-g\right] / 2\right)
\end{aligned}
$$


In the last equation of (6), we have taken into account that the magnon damping is proportional to $g^{2}, \bar{\gamma}=k g^{2} \bar{\omega}$ (the factor $k$ is determined by the bandstructure and magnetic ordering), and the scaling equations should contain only renormalized quantities.

\section{THE SCALING BEHAVIOR IN THE PRESENCE OF DAMPING}

As demonstrated in Ref. [15], the singularities of the scaling function $\eta$ can result in occurrence of a NFL behavior in a restricted region due to fixing of the argument of the function $\eta$ at the singularity during the scaling process, so that $\bar{\omega}(C) \simeq|C|$,

$$
g_{e f}(\xi)-g \simeq 2(\xi-\lambda) / a, \lambda \equiv \ln (D / \bar{\omega}),
$$

(the scale $D$ is defined by $g_{e f}(-D)=g, \xi \equiv \ln |D / C|$ ). This region becomes not too narrow only provided that the bare coupling constant $g=-2 I \rho$ is very close to the critical value $g_{c}$ for the magnetic instability $\left(\left|g-g_{c}\right| / g_{c} \sim 10^{-4} \div 10^{-6}\right)$. Here we consider the scaling process with account of a not too small magnon damping (only very small damping was introduced in Ref. [15] to provide existence of the magnetic-non-magnetic ground-state transition at $\left.g=g_{c}\right)$

The scaling function $\eta$ in the FM and AFM phases for simple magnetic structures reads (for simplicity, magnetic anisotropy is neglected in this Section)

$$
\eta\left(\bar{\omega}_{e x} /|C|, \bar{\gamma} /|C|\right)=\operatorname{Re}\left\langle\left(1-\left(\omega_{\mathbf{k}-\mathbf{k}^{\prime}}+i \gamma_{\mathbf{k}-\mathbf{k}^{\prime}}\right)^{2} / C^{2}\right)^{-1}\right\rangle_{t_{k}=t_{k^{\prime}}=0}
$$

For an isotropic three-dimensional (3D) ferromagnet integration in (8) for $\gamma=$ const and quadratic spin-wave spectrum yields

$$
\eta(x, z)=\frac{1+z^{2}}{4 x} \ln \frac{(1+x)^{2}+z^{2}}{(1-x)^{2}+z^{2}}+\frac{1+x}{z} \arctan \frac{z}{1+x}-\frac{1-x}{z} \arctan \frac{z}{1-x}
$$

where $x=\bar{\omega}_{e x} /|C|, z=\bar{\gamma} /|C|$. Note that last two terms in (9) play a role similar to that of the "incoherent" contribution to the function $\eta$, which was treated in Ref. 15.

For a 3D antiferromagnet integration in (8) for the linear spin-wave spectrum gives 


$$
\eta(x, z)=\frac{1}{2} \operatorname{Re}[(1+i z) \ln (1+x+i z)+(1-i z) \ln (1+x-i z)]
$$

where we take into account the intersubband damping only,

$$
\begin{aligned}
& \gamma_{\mathbf{q}}=\pi 2 I^{2} \bar{S}\left(J_{\mathbf{q}}-J_{\mathbf{Q}}\right) \rho^{2} \lambda_{\mathbf{q}+\mathbf{Q}} \\
& \lambda_{\mathbf{q}}=\rho^{-2} \sum_{\mathbf{k}} \delta\left(t_{\mathbf{k}}\right) \delta\left(t_{\mathbf{k}+\mathbf{q}}\right) .
\end{aligned}
$$

The damping (11) can be put nearly constant at not too large $q$ (the threshold value determined by the AFM gap can be neglected due to formal smallness in $I$ ). In the $2 \mathrm{D}$ case we obtain in the same approximation

$$
\begin{aligned}
\eta(x, z) & =\frac{\nu^{3}(x, z)}{\nu^{4}(x, z)+z^{2}} \\
\nu^{2}(x, z) & =\frac{1}{2}\left[1-x^{2}+\sqrt{\left(1-x^{2}\right)^{2}+4 z^{2}}\right]
\end{aligned}
$$

which modifies somewhat the result of Ref. [15.

The function $\lambda_{\mathbf{q}}$ determines, in particular, the factor $k$ in (6). For a parabolic electron spectrum we obtain

$$
\lambda_{\mathbf{q}}=\frac{\theta(1-x)}{z x} \times \begin{cases}1 / 6, & d=3 \\ (4 \pi)^{-1}\left(1-x^{2}\right)^{-1 / 2}, & d=2\end{cases}
$$

where $x=q / 2 k_{F}, \theta(x)$ is the step function, $z$ is the electron concentration (with both spin projections).

Main Kondo renormalization of the magnon damping comes from its proportionality to the factor of $\bar{S}$. Spin fluctuations can give correction to this factor, as well as for the magnon frequency [15]. For simplicity, we restrict ourselves in numerical calculations to the AFM case with $\delta \gamma_{\mathbf{q}} / \gamma_{\mathbf{q}}=\delta \omega_{\mathbf{q}} / \omega_{\mathbf{q}}=\delta \bar{S} / S$, so that $c=1$ in (幽, (6). The corresponding scaling trajectories are shown in Fig.1. One can see that, unlike Ref. [15], the "linear" behavior, although being somewhat smeared, is pronounced in a considerable region of $\xi$ for not too small $\left|g-g_{c}\right|$, especially in the 2D case. In the 3D case the linear region (7) is followed by a quasi-linear behavior with 


$$
g_{e f}(\xi) \simeq A(\xi-\lambda), \bar{\omega}(C) \propto|C|^{a A / 2}, \bar{S}_{e f}(C) \propto|C|^{A / 2}
$$

where $A<2 / a$. To investigate the latter behavior in more details, it is instructive to consider also the case of a paramagnet with pure dissipative dynamics. In the case of spin-diffusion behavior we have (cf. Ref. [15])

$$
\eta^{P M}\left(\frac{\bar{\omega}}{C}\right)=\sum_{\mathbf{q}} \lambda_{\mathbf{q}}\left[1+\left(\mathcal{D} q^{2} / C\right)^{2}\right]^{-1}, \bar{\omega}=4 \mathcal{D} k_{F}^{2}
$$

where $\mathcal{D}$ is the spin diffusion constant. As demonstrate numerical calculations (see Fig.2), for $g \leq g_{c}$ the one-impurity behavior $1 / g_{e f}(\xi)=1 / g-\xi$ is changed at $\xi \simeq \lambda$ by the behavior (15) with

$$
A \simeq\left[g_{e f}(\lambda)\right]^{2} \Psi(0), g_{e f}(\lambda) \simeq g /(1-\lambda g), \Psi(0)=\eta(1) \sim 0.5
$$

In the 3D case where $\eta^{P M}(x)=\arctan x / x$ the quasi-linear NFL-like behavior $g_{e f}(\xi)$ takes place in a rather narrow region. However, in the 2D case we obtain

$$
\eta^{P M}(x)=\left[\frac{1+\left(1+x^{2}\right)^{1 / 2}}{2\left(1+x^{2}\right)}\right]^{1 / 2}
$$

and the NFL-like region becomes more wide due to a more slow decrease of $\eta^{P M}(x)$ at $x \rightarrow \infty$. Note that such regions are not observed for $g>g_{c}$.

\section{SINGULARITIES OF THE SCALING FUNCTION}

To get further insight into the NFL-behavior problem, we perform an analysis of singularities of the scaling function $\eta$, which is more general than in Ref. [15. In the absence of damping we can write down

$$
\eta\left(\bar{\omega}_{e x} /|C|, \omega_{0} /|C|\right)=\sum_{\mathbf{q}} \lambda_{\mathbf{q}}\left(1-\omega_{\mathbf{q}}^{2} / C^{2}\right)^{-1}
$$

The singularities of $\eta$ correspond to the Van Hove singularities in the magnon spectrum and to the boundary points $q=0$ and $q=2 k_{F}$.

For $q \rightarrow 2 k_{F}$ the magnon spectrum has the Kohn anomaly 


$$
\delta \omega_{\mathbf{q}} \propto\left\{\begin{array}{l}
\left(q-2 \dot{k}_{F}\right) \ln \left|q-2 k_{F}\right|, d=3 \\
\sqrt{q^{2}-4 k_{F}^{2}} \theta\left(q-2 k_{F}\right), d=2
\end{array}\right.
$$

Taking into account the dependences (14), which hold qualitatively in a general situation, we obtain for $v=C^{2}-\bar{\omega}^{2} \rightarrow 0$

$$
\eta(v) \propto\left\{\begin{array}{l}
\ln \ln |v|, \quad d=3 \\
\theta(v) v^{-1 / 2}, \quad d=2
\end{array}\right.
$$

Note that the singularity of the form $\ln v$ obtained for the $3 D$ case in Ref. [15] (see also previous Section), is a consequence of a simplified "Debye" model of the magnon spectrum. In fact, such a dependence corresponds qualitatively to an intermediate asymptotics at approaching the singularity. In any case, a small damping of spin excitations should be introduced to cut the singularity. In the calculations below we put the damping parameter $\delta=1 / 100$ (see Ref. [15]). In fact, pronounced extrema of $\eta$, but not singularities themselves turn out to be important for the scaling behavior discussed below.

For $q \rightarrow 0$ we have $\lambda_{\mathbf{q}} \propto q^{-1}$. Near the points of minimum (maximum) in the magnon spectrum we have $\omega_{\mathbf{q}}^{2}-\omega_{m}^{2} \propto \pm q^{2}$, and for $v=C^{2}-\omega_{m}^{2} \rightarrow 0$ we obtain

$$
\eta(v) \propto \begin{cases} \pm \ln |v|, & d=3 \\ \mp \theta(\mp v)|v|^{-1 / 2}, & d=2\end{cases}
$$

so that $\eta \rightarrow-\infty$ near the band bottom and $\eta \rightarrow+\infty$ near the band top. The Van Hove singularities in the magnon band at $\omega=\omega_{c}$ for $q \neq 0$ yield weaker singularities in $\eta(v)\left(|v|^{1 / 2}\right.$ for $d=3$ and a finite jump for $d=2$ ) and will not be treated below.

\section{THE SCALING PICTURE AND NFL BEHAVIOR IN MANY-SUBLATTICE MAGNETS}

Using the results of the previous Section we can propose a rather realistic and universal mechanism of the NFL behavior. Suppose that the spin excitation spectrum contains several branches which make additive contributions to the function $\eta$. 
As a simple example we can consider a two-sublattice ferrimagnet with the localizedsystem Hamiltonian

$$
H_{f}=\sum_{\mathbf{q}}\left(J_{\mathbf{q}} \mathbf{S}_{-\mathbf{q}} \mathbf{S}_{\mathbf{q}}+J_{\mathbf{q}}^{\prime} \mathbf{S}_{-\mathbf{q}}^{\prime} \mathbf{S}_{\mathbf{q}}^{\prime}+\widetilde{J}_{\mathbf{q}} \mathbf{S}_{-\mathbf{q}} \mathbf{S}_{\mathbf{q}}^{\prime}\right)
$$

the $s-f$ exchange interaction being taken into account only at one sublattice (spins without primes). Similar to (19) we obtain

$$
\eta=\sum_{\mathbf{q}, i=1,2} \lambda_{\mathbf{q}} \frac{\omega_{\mathbf{q} i}}{B_{\mathbf{q}}}\left(1-\frac{\omega_{\mathbf{q} i}^{2}}{C^{2}}\right)^{-1}
$$

where

$$
\begin{aligned}
B_{\mathbf{q}} & =\left\{\left[S\left(J_{\mathbf{q}}-J_{0}\right)+S^{\prime}\left(J_{\mathbf{q}}^{\prime}-J_{0}^{\prime}\right)-\left(S+S^{\prime}\right) \widetilde{J}_{0}\right]^{2}-4 S S^{\prime} \widetilde{J}_{\mathbf{q}}^{2}\right\}^{1 / 2}, \\
\omega_{\mathbf{q} 1,2} & =B_{\mathbf{q}} \mp\left|S\left(J_{\mathbf{q}}-J_{0}\right)-S^{\prime}\left(J_{\mathbf{q}}^{\prime}-J_{0}^{\prime}\right)+\left(S-S^{\prime}\right) \widetilde{J}_{0}\right|
\end{aligned}
$$

are the acoustical and optical modes.

The dependence

$$
\Psi(\xi)=\sum_{i} z_{i} \eta_{i}\left[\left(\bar{\omega}_{e x, i} / D\right) e^{\xi},\left(\omega_{0, i} / D\right) e^{\xi}\right]
$$

is shown in Fig.3 for the case of two excitation modes in a 3D antiferromagnet (the expressions for the function $\eta_{i}$ in the case of one mode with inclusion of anisotropy are given in Ref. [16]). The only property of the function $\Psi$, which will be important below, is the occurrence of the second zero with decreasing $|C|$ (or increasing $\xi$ ). This property follows immediately from existence of the "positive" singularity in $\Psi$ near the maximum frequency of the lower branch and of the "negative" singularity near the minimum frequency of the upper branch (e.g., for $\left.\omega_{1}\left(q=2 k_{F}\right)<\omega_{2}(q=0)\right)$. One can expect that this is a general property of manysublattice magnets. The singularities can be also connected with the crystal-field excitations [3.

As demonstrate both numerical calculations and analytical treatment, in some interval of the bare coupling parameter, $g_{c 1}<g<g_{c 2}$, the argument of the function $\Psi$ becomes fixed at the second zero, $C=C_{0}$, during the scaling process which is described by Eq.(2)). This can 
be illustrated for the simple case where $a=b$ for all the modes (e.g., for an antiferromagnet in the nearest-neighbor approximation we have $a=b=1$ ). On substituting (6) into (2) we obtain

$$
\partial\left(1 / g_{e f}\right) / \partial \xi=-\Psi\left(\xi-a\left[g_{e f}-g\right] / 2\right)
$$

Then we derive

$$
g_{e f}(\xi \rightarrow \infty) \simeq(2 / a)\left(\xi-\xi_{0}\right)-1 /\left[\Psi^{\prime}\left(\xi_{0}\right) \xi^{2}\right]
$$

where $\xi_{0} \sim \ln (D / \bar{\omega})$ is the second zero of the function $\Psi(\xi)$. Note that the first zero does not work since the unrestricted increase of $g_{\text {ef }}$ corresponds to a decrease of the argument of the function $\Psi$ in (27), so that $\Psi \rightarrow+0$. According to (6) we obtain

$$
\bar{\omega}_{e x}(C), \omega_{0}(C) \propto|C|, \bar{S}_{e f}(C) \propto|C|^{1 / a}
$$

Within the approach used, the behavior (28),(29) takes place up to $C=0(\xi=\infty)$. Although the one-loop scaling equations themselves may become invalid with increasing $g_{e f}$, the tendency to the formation of the "soft" magnon mode seems to be physically correct. The scaling picture for three possible cases is shown in Fig.4. One can see that the interval $\left[g_{c 1}, g_{c 2}\right]$ where the NFL behavior occurs is not too small, unlike Ref. [15].

In a more general case, where $a \neq b$ and the exponents in (3) differ for different frequencies, the linear $C$-dependence takes place only for the total characteristic frequency $\bar{\omega}$ (e.g., for an anisotropic antiferromagnet with one mode we have $\left.\bar{\omega}^{2}=\omega_{0}^{2}+\bar{\omega}_{e x}^{2}\right)$, and the behavior

$g_{e f}(\xi)$ and $\bar{S}_{e f}(\xi)$ is more complicated. As follows from (5), for the AFM state with small next-nearest exchange interactions the case of small $|a-1|$ and $b=1$ is realized.

\section{DISCUSSION OF PHYSICAL PROPERTIES AND CONCLUSIONS}

Consider the temperature dependence of the magnetic susceptibility. In the spin-wave region we have for an AFM structure with the wavevector $\mathbf{Q}$ 


$$
\chi=\lim _{q \rightarrow 0}\left\langle\left\langle S_{\mathbf{q}}^{x} \mid S_{-\mathbf{q}}^{x}\right\rangle\right\rangle_{\omega=0}=\left(J_{0}-J_{\mathbf{Q}}\right)^{-1} \propto \bar{S} / \bar{\omega}
$$

One can assume that the spin-wave description of the electron-magnon interaction is adequate not only in the AFM phase, but also for systems with a strong short-range AFM order (e.g., for 2D and frustrated 3D systems at finite temperatures). Anomalous $f$-systems demonstrate indeed pronounced quasi-2D spin fluctuations, see, e.g., Refs. [14,18. Using the scaling arguments we can replace $\bar{\omega} \rightarrow \bar{\omega}(C), \bar{S} \rightarrow \bar{S}_{e f}(C)$ with $|C| \sim T$, which yields

$$
\chi(T) \propto T^{-\zeta}, \zeta=(a-1) / a
$$

According to (5), the non-universal exponent $\lambda$ is determined by details of magnetic structure and can be both positive and negative. For a qualitative discussion, we can still use Fig.4 and treat the difference $a-1$ as a perturbation. The increase of $\chi(T \rightarrow 0)$ (which is usually called NFL behavior) takes place for $a>1$ and, as follows from (6), corresponds to an increase of magnetic anisotropy parameter with lowering $T$ (see Ref. [16]). Such a correlation may be verified experimentally.

The temperature dependence of electronic specific heat can be estimated from the secondorder perturbation theory, $C_{e l}(T) / T \propto 1 / Z(T)$ where $Z(T)$ is the residue of the electron Green's function at the distance $T$ from the Fermi level (cf. Ref. [17]). Then we have

$$
C_{e l}(T) / T \propto g_{e f}^{2}(T) \bar{S}_{e f}(T) / \bar{\omega}_{e x}(T) \propto \chi(T) \ln ^{2} T
$$

The dependence $C_{e l}(T) / T \propto \chi(T)$ has been recently obtained experimentally for a wide class of NFL systems [11]. One can expect that the accuracy of the experimental data is insufficient to pick out the factor of $\ln ^{2} T$. Generally, the temperature behavior of magnetic characteristics $(\bar{S}$ and $\bar{\omega})$, which depend exponentially on the coupling constant, is decisive for our NFL mechanisms. The transport relaxation rate determining the temperature dependence of the resistivity owing to scattering by spin fluctuations in AFM phase is given by 19

$$
\frac{1}{\tau}=\frac{2 \pi}{v_{F}^{2}} I^{2} \bar{S}^{2}\left(J_{0}-J_{\mathbf{Q}}\right) \rho\left\langle\left(\mathbf{v}_{\mathbf{k}+\mathbf{Q}}-\mathbf{v}_{\mathbf{k}}\right)^{2}\right\rangle_{t_{\mathbf{k}}=0} \sum_{\mathbf{q} \simeq \mathbf{Q}} \lambda_{\mathbf{q}}\left(-\frac{\partial N_{\mathbf{q}}}{\partial \omega_{\mathbf{q}}}\right)
$$


Then we obtain

$$
\frac{1}{\tau} \propto g_{e f}^{2}(T) \bar{S}_{e f}(T) / \bar{\omega}_{e x}(T) T^{2} \propto T^{2} C_{e l}(T) / T \propto T^{2-\zeta}
$$

Considering electron-electron scattering as the main scattering mechanism one can expect another temperature dependence, namely

$$
\frac{1}{\tau} \propto[\rho / Z(T)]^{2} T^{2} \propto T^{2}\left[C_{e l}(T) / T\right]^{2} \propto T^{2-2 \zeta}
$$

It would be interesting to compare experimental dependences on one hand, of the resistivity and, on the other hand, of the susceptibility and specific heat, to choose between dependences (34) and (35). Validity of the relation (34) would be a verification of the scenario for NFL state formation proposed here.

The temperature dependences of specific heat, magnetic susceptibility and resistivity in the case of a NFL-like behavior considered in Sect.3 differ from those discussed above by the value of $\zeta=(a-1) A / 2$ (for the second "linear" region).

To conclude, phenomenon of the NFL behavior seems to have a complicated nature, so that it is hardly possible to propose an unified picture for all the cases. The mechanisms considered above are not based on disorder effects, but describe naturally the NFL state in ideal crystals. At the same time, the damping makes the quasi-NFL behavior considered in Sect.3 more pronounced and in a sense plays the role of disorder.

The damping is not important for the NFL behavior mechanism considered in Sect.5. This NFL picture is "true" (i.e. holds up to lowest temperatures) within the lowest-order scaling approach; treatment of higher-order corrections to the scaling equations would provide additional information. Unlike previous phenomenological works, existence of peculiar long-range critical fluctuations near the quantum phase transition is not needed for this mechanism, but local reconstruction of electronic states owing to the Kondo effect is essential, the concrete form of spin spectral function being of crucial importance. More detailed investigations of the NFL behavior for complicated spectral functions, in particular with account of incoherent contributions, would be also of interest. 
The research described was supported in part by Grant No.99-02-16279 from the Russian Basic Research Foundation. 


\section{Figure CAPTiOnS}

Fig.1. The scaling trajectories $g_{e f}(\xi)$ in isotropic 2D antiferromagnets (solid lines, $g=$ $0.154<g_{c}, g=0.155>g_{c}$ ) and 3D antiferromagnets (dashed lines, $g=0.139<g_{c}, g=$ $\left.0.140>g_{c}\right)$ with $k=0.5, a=c=1, \lambda=\ln (D / \bar{\omega})=5$.

Fig.2. The scaling trajectories $g_{e f}(\xi)$ in 2D (solid lines) and 3D (dashed lines) paramagnets with $a=1 / 2, \lambda=5$. The bare coupling parameters are $g=0.135$ and $g=0.145$, higher curves corresponding to larger $g$. The values of $g_{c}$ in 2D and 3D cases are 0.148 and 0.152 .

Fig.3. The scaling functions $\Psi(\xi)$ defined by (26) in the case of a 3D antiferromagnet with two excitation modes The parameters are $z_{1}=0.4, z_{2}=0.6, \ln \left(D / \bar{\omega}_{2}\right)=4, \bar{\omega}_{2} / \bar{\omega}_{1}=3$, $\omega_{0,1} / \bar{\omega}_{e x, 1}=0.2, \omega_{0,2} / \bar{\omega}_{e x, 2}=0.6\left(\bar{\omega}_{i}^{2}=\bar{\omega}_{e x, i}^{2}+\omega_{0, i}^{2}\right)$.

Fig.4. The scaling trajectories $g_{e f}(\xi)$ in a 3D antiferromagnet with the parameters of Fig. 3 and $a_{i}=b_{i}=1$. The bare coupling parameters are $g=0.158 \simeq g_{c 2}$ (upper solid line

with the asymptotics), $g=0.154 \simeq g_{c 1}$ (lower solid line), and $g_{c 1}<g=0.156<g_{c 2}$ (dashed line). 


\section{REFERENCES}

* E-mail: Valentin.Irkhin@imp.uran.ru

[1] M.B. Maple et al, J.Low Temp.Phys.95, 225 (1994); 99, 223 (1995); Ref. [2], p.9773.

[2] Proc.Conf. Non-Fermi-Liquid Behavior in Metals (Santa-Barbara, 1996), J.Phys.:Cond.Mat.8, p.9675-10148 (1996).

[3] P. Fulde and M. Löwenhaupt, Adv.Phys.34, 589 (1986).

[4] K.Umeo, H. Kadomatsu, and T. Takabatake, Ref. [2], p.9743.

[5] F. Steglich et al, Ref. [2], p.9909.

[6] It should be noted that a possibility of a NFL behavior of transport properties in disordered systems has been discussed many years ago in the paper by B.L. Altshuler and A.G. Aronov, Zh.Exp.Theor.Fiz.77, 2028 (1979).

[7] B. Andraka and A.M. Tsvelik, Phys.Rev.Lett.67, 2886 (1991); A.M. Tsvelick and M. Rivier, Phys.Rev.B48, 9887 (1993).

[8] D.L. Cox and M. Jarrell, J.Phys.:Cond.Mat.8, 9825 (1996).

[9] P. Coleman, L.B. Ioffe, and A.M. Tsvelik, Phys.Rev.B52, 6611 (1995).

[10] A.H. Castro-Neto, G. Castilla, and B.A. Jones, Phys.Rev.Lett.81, 3531 (1998).

[11] M.C. Andrade et al, Phys.Rev.Lett.81, 5620 (1998).

[12] S.R. Julian et al, Ref. [2], p.9675; A. Schröder et al, Phys.Rev.Lett.80, 5623 (1998).

[13] L.B. Ioffe and A.J. Millis, Usp.Fiz.Nauk 168, 672 (1998).

[14] P. Coleman, cond-mat/9809436.

[15] V.Yu. Irkhin and M.I. Katsnelson, Phys.Rev.B56, 8109 (1997);

[16] V.Yu. Irkhin and M.I. Katsnelson, Phys.Rev.B59, 9348 (1999). 
[17] V.Yu. Irkhin and M.I. Katsnelson, Z.Phys.B75, 67 (1989).

[18] O. Stockert et al, Phys.Rev.Lett.80, 5627 (1998).

[19] V.Yu. Irkhin and M.I. Katsnelson, Phys.Rev.B52, 6181 (1995). 


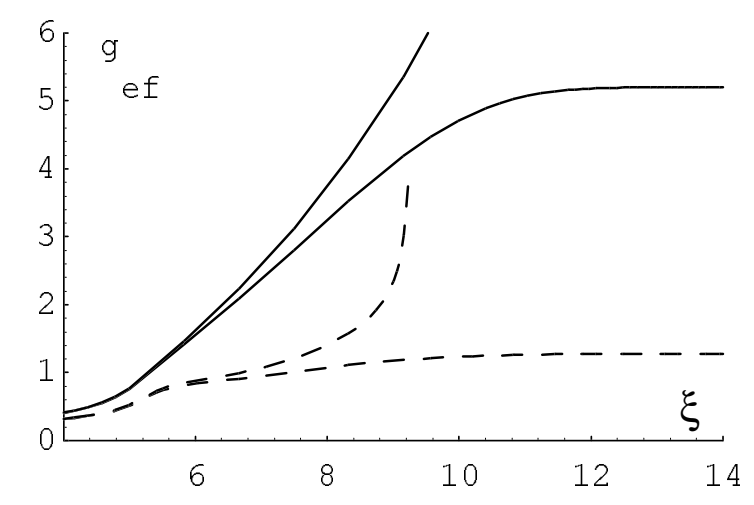




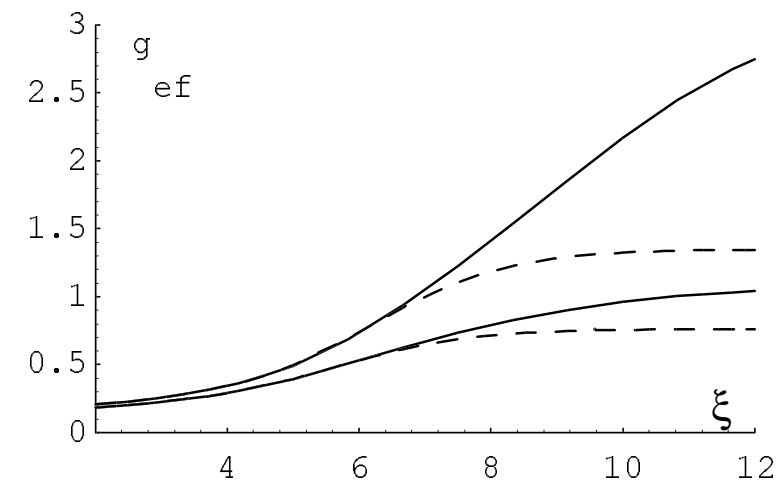




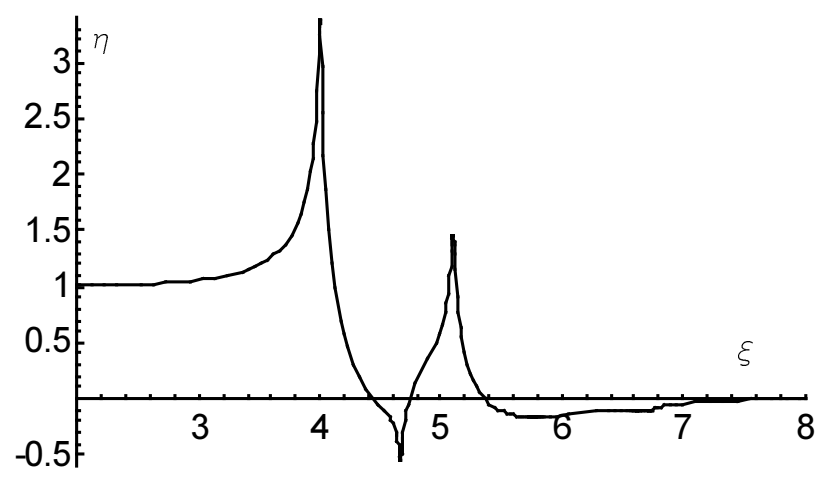




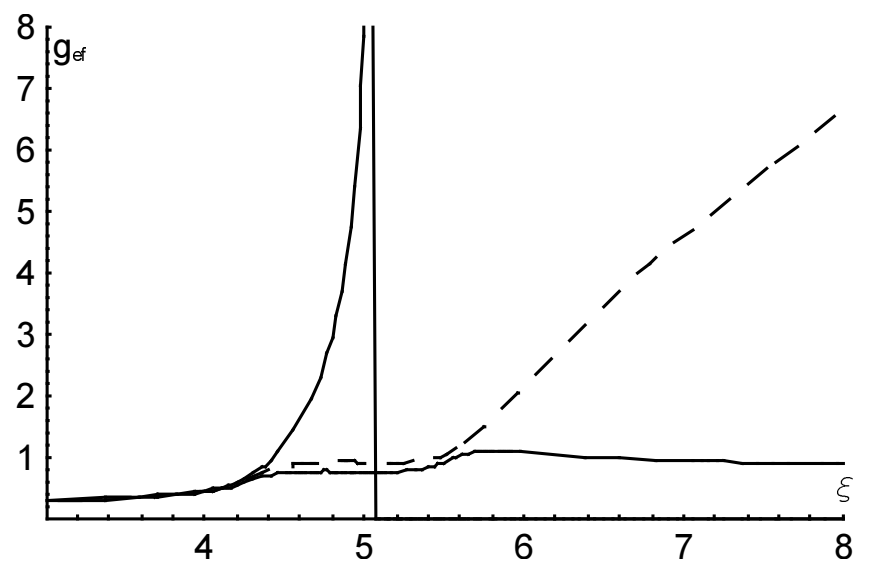

